\title{
Twenty four hour variation in plasma atrial natriuretic factor during VVI and DDD pacing
}

Panagiotis E Vardas, Manolis Markianos, Emmanuel Skalidis, Emmanuel Simantirakis, Emmanuel Manios, Efstathios Papavasiliou

\begin{abstract}
Objective-To investigate whether plasma atrial natriuretic factor (ANF) follows a pattern of circadian variation similar to that of other hormones in patients paced under VVI and DDD pacing modes and to determine if the known effect of pacing mode on ANF secretion is maintained throughout the 24 hour period.
\end{abstract}

Patients and Design-20 patients were studied. They had complete atrioventricular block and had been paced for 17 (SD 3.5) months with a dual chamber multiprogrammable pacemaker. They were divided into two groups according to the duration of pacing in either VVI or DDD mode before the measurements: group $A$, $n=11$ (8 men, 3 women, aged 65 (7) years), each paced for $24 \mathrm{~h}$ under each of VVI and DDD modes in random order; group $B, n=9$ ( 7 men, 2 women, aged 63 (8) years), each paced for $60 \mathrm{~d}$ under each pacing mode before the measurements. Blood samples were taken and ANF concentrations measured every $4 \mathrm{~h}$ over a $24 \mathrm{~h}$ period, starting at 09.00. Measurements were also made of plasma cortisol, which has a known circadian pattern, so that the $24 \mathrm{~h}$ curve could be compared with that of ANF.

Results-In contrast to cortisol, ANF values indicated a pulsatile pattern of secretion throughout the $24 \mathrm{~h}$ period, with no clear circadian variation. In group B, ANF concentrations were significantly higher during VVI than during DDD pacing throughout the $24 \mathrm{~h}$ period, whereas in group $\mathbf{A}$ this difference was statistically significant only at certain times of day. Conclusions-ANF does not show the circadian pattern of variation shown by cortisol and other hormones. Dual chamber pacing contributes to an improvement not only in cardiac haemodynamics but also in the neuroendocrine system, especially in the long term.

(Heart 1996;75:620-622)

Keywords: atrial natriuretic factor, dual chamber pacing, ventricular pacing, circadian variation.

Several studies have established that the pacing mode affects not only the patient's haemodynamic state but also the neuroendocrine system. In particular, plasma atrial natriuretic factor (ANF) appears to be especially influenced by pacing mode, increasing during VVI pacing and rapidly returning to normal following the restoration of the atrioventricular sequence, as happens with dual chamber pacing. Although these observations have been well reported previously ${ }^{12}$ some important related questions remain to be elucidated. Among these are the following: (1) it is not clear if the observed differences in plasma ANF between VVI and DDD pacing are maintained throughout a 24 hour period; (2) it is not clear whether plasma levels of atrial natriuretic factor follow a circadian pattern, as happens with other hormones, especially in patients paced in VVI and DDD pacing modes; (3) it is not known if the duration of pacing, with either VVI or DDD mode, has an effect on the above findings. The purpose of this study was to investigate these questions.

\section{Methods}

We studied 20 patients with complete atrioventricular block who had been paced for 17 (SD 3.5) months with a dual chamber multiprogrammable pacemaker. All patients had normal atrial activity and none had retrograde atrioventricular conduction. Exclusion criteria were kidney failure, heart failure, and age over 75 years. For the evaluation of heart failure, apart from the clinical criteria, each patient underwent a detailed echocardiographic study which included the measurement of systolic and diastolic indices of left ventricular function.

The 20 patients were divided into two groups which differed only according to the duration of pacing before the start of measurements. (1) Group A (11 patients, eight men and three women, aged 65 (7) years): each patient was paced for $24 \mathrm{~h}$ under VVI or DDD pacing mode in random order, and with a set lower pacing rate of 70 beats $/ \mathrm{min}$ in both pacing modes. The pacing mode was programmed $2 \mathrm{~h}$ before the first blood sample was taken, in order to allow time for the levels of ANF to adjust. (2) Group B (nine patients, seven men and two women, aged 63 (8) years): in this group the patients were paced for $60 \mathrm{~d}$ before the first blood sample was taken. As with group $A$, the patients were paced under both VVI and DDD modes in random order and with a set lower pacing rate of 70 beats/min in both pacing modes.

The atrioventricular delay in both groups of patients was programmed on the basis of the echocardiographic evaluation so that each
University Hospital of Crete, Greece.

Accepted for publication 13 November 1995 
(A) Mean values (standard deviation in parentheses) of atrial natriuretic factor (ANF) and cortisol plasma levels in samples taken every four hours during VVI and DDD pacing in a cross over design with $24 \mathrm{~h}$ (group $A$ ) and 2 month (group B) duration of pacing under each mode before measurements

\begin{tabular}{|c|c|c|c|c|c|c|c|c|}
\hline \multirow[b]{3}{*}{ Time } & \multicolumn{4}{|l|}{ Group $A$} & \multicolumn{4}{|l|}{ Group B } \\
\hline & \multicolumn{2}{|c|}{$A N F(f m o l / m l)$} & \multicolumn{2}{|c|}{ Cortisol (ng/ml) } & \multicolumn{2}{|c|}{$A N F($ fmol/ml) } & \multicolumn{2}{|c|}{ Cortisol (ng/ml) } \\
\hline & $V V I$ & $D D D$ & $V V I$ & $D D D$ & $V V I$ & $D D D$ & $V V I$ & $D D D$ \\
\hline $\begin{array}{l}09: 00 \\
13: 00 \\
17: 00 \\
21: 00 \\
01: 00 \\
05: 00\end{array}$ & $\begin{array}{l}88 \cdot 2(21 \cdot 3) \\
87 \cdot 2(20 \cdot 0) \\
89 \cdot 7(17 \cdot 5) \\
87 \cdot 6(21 \cdot 3) \\
87 \cdot 7(24 \cdot 6) \\
84 \cdot 2(18 \cdot 6)\end{array}$ & $\begin{array}{l}80 \cdot 1(10 \cdot 9) \\
79 \cdot 7(11 \cdot 2) \\
80 \cdot 4(15 \cdot 4) \\
77 \cdot 7(14 \cdot 7) \\
76 \cdot 8(14 \cdot 2) \\
75 \cdot 7(11 \cdot 9)\end{array}$ & $\begin{array}{l}99 \cdot 7(34.4)^{\star} \\
78.3(21.5) \\
66.3(25.6) \\
40.0(10.4)^{\star} \\
38.6(12.9)^{\star} \\
87.3(30.4)\end{array}$ & $\begin{array}{c}105.0(25.5)^{\star} \\
65.1(10.4)^{\star} \\
51.4(13.4)^{\star} \\
36.9(9.0)^{\star} \\
43.8(24.2) \\
73.5(39.7)\end{array}$ & $\begin{array}{l}81 \cdot 9(24 \cdot 5) \\
86 \cdot 8(24 \cdot 0) \\
81 \cdot 5(21 \cdot 2) \\
85 \cdot 8(22 \cdot 6) \\
84 \cdot 1(25 \cdot 2) \\
83.5(18 \cdot 9)\end{array}$ & $\begin{array}{l}66 \cdot 1(10 \cdot 8) \\
64 \cdot 7(9 \cdot 6) \\
65 \cdot 4(11 \cdot 1) \\
66 \cdot 2(9 \cdot 1) \\
64 \cdot 8(7 \cdot 9) \\
64 \cdot 2(9 \cdot 8)\end{array}$ & $\begin{array}{c}131(25)^{\star} \\
131(73)^{\star} \\
68(21)^{\star} \\
63(35)^{\star} \\
83(44) \\
143(46)\end{array}$ & $\begin{array}{c}139(44)^{\star} \\
124(48) \\
76(44)^{\star} \\
84(61) \\
59(33) \\
167(63)^{\star}\end{array}$ \\
\hline Mean & $87 \cdot 4(19 \cdot 6)$ & $78.4(11.8)$ & $68 \cdot 5(11 \cdot 5)$ & $62 \cdot 7(9 \cdot 8)$ & $83.9(21 \cdot 1)$ & $65 \cdot 2(8 \cdot 4) \dagger$ & $103(26)$ & $108(36)$ \\
\hline
\end{tabular}

*Significantly different after Bonferroni correction compared to the mean of the six measurements.

†Significantly different compared to the VVI mean.

(B) Repeated measures analysis of variance

\begin{tabular}{|c|c|c|c|c|c|c|c|c|}
\hline \multirow[b]{2}{*}{ Effect } & \multicolumn{2}{|l|}{$A N F$} & \multicolumn{2}{|c|}{ Cortisol } & \multicolumn{2}{|l|}{$A N F$} & \multicolumn{2}{|c|}{ Cortisol } \\
\hline & $F$ & $P$ & $F$ & $P$ & $F$ & $P$ & $F$ & $P$ \\
\hline $\begin{array}{l}\text { Pacing } \\
\text { Time } \\
\mathrm{P} \times \mathrm{T}\end{array}$ & $\begin{array}{l}1 \cdot 72 \\
1.48 \\
0 \cdot 17\end{array}$ & $\begin{array}{l}0 \cdot 20 \\
0 \cdot 20 \\
0 \cdot 97\end{array}$ & $\begin{array}{r}1.60 \\
24.64 \\
0.93\end{array}$ & $\begin{array}{l}0.22 \\
0.00 \\
0.46\end{array}$ & $\begin{array}{l}6 \cdot 07 \\
0 \cdot 32 \\
0 \cdot 42\end{array}$ & $\begin{array}{l}0.025 \\
0.89 \\
0.83\end{array}$ & $\begin{array}{r}0.13 \\
18.32 \\
1.08\end{array}$ & $\begin{array}{l}0.73 \\
0.00 \\
0.37\end{array}$ \\
\hline
\end{tabular}

individual patient should have the best possible haemodynamic response.

Blood samples were taken for ANF measurement every $4 \mathrm{~h}$ over a $24 \mathrm{~h}$ period, starting at $09 \cdot 00$. During the period $09 \cdot 00-21 \cdot 00$, when the patients were awake and mobile, they lay down for $30 \mathrm{~min}$ before a blood sample was taken. Measurements were also made of plasma cortisol, a hormone with a known circadian pattern, so that the $24 \mathrm{~h}$ curve could be compared with that of ANF.

Plasma ANF was measured by radioimmunoassay (Amersham) and expressed in $\mathrm{fmol} / \mathrm{ml}$. We applied the procedure without extraction, since preliminary experiments with the use of columns gave ANF concentrations similar to those obtained without extraction for samples with concentrations below 640 $\mathrm{fmol} / \mathrm{ml}$, while the coefficients of variation were lower without extraction. The coefficients of variation were around $15 \%$.

Serum cortisol was also measured by radioimmunoassay (Sorin Biomedica). The within and between assay coefficients of variation were below $10 \%$. Concentrations were expressed in $\mathrm{ng} / \mathrm{ml}$.

\section{STATISTICAL ANALYSIS}

We used repeated measures analysis of variance to search for possible effects of pacing mode and time on the concentrations of plasma ANF and cortisol. When a pacing effect was found, the mean values of the six measurements for VVI and DDD modes were compared. In the case of a significant time effect, the concentrations at each time point were compared to the mean of the six measurements in order to identify the acrophases of the variation, taking as significance level $P<0.008$ (Bonferroni correction).

\section{Results}

The table shows the mean values of plasma ANF and cortisol in samples taken every $4 \mathrm{~h}$ during VVI and DDD pacing in crossover design, with $24 \mathrm{~h}$ (group A) and two month (group B) duration of each pacing mode.

In the patients in group $A$, who were paced for $24 \mathrm{~h}$ in each pacing mode, the ANF concentrations were higher under VVI mode than under DDD mode only at certain times, and no significant overall pacing effect was found $(F=1 \cdot 72, P=0.20)$. A clear pacing effect was found in the second group of patients ( $F$ $=6.07, P=0.025$ ), where the patients were paced for two months before measurements in each mode.

No time effect was found in either group of patients for ANF (table) under either VVI or DDD pacing mode. In contrast, plasma cortisol showed a significant fall at $17 \cdot 00,21 \cdot 00$, and 01.00 and an increase at 05.00 and 09.00 , compared with the mean value for the day, under both pacing modes (figure). This showed that cortisol follows its known circadian pattern in paced patients, and it confirmed the reliability of the methods

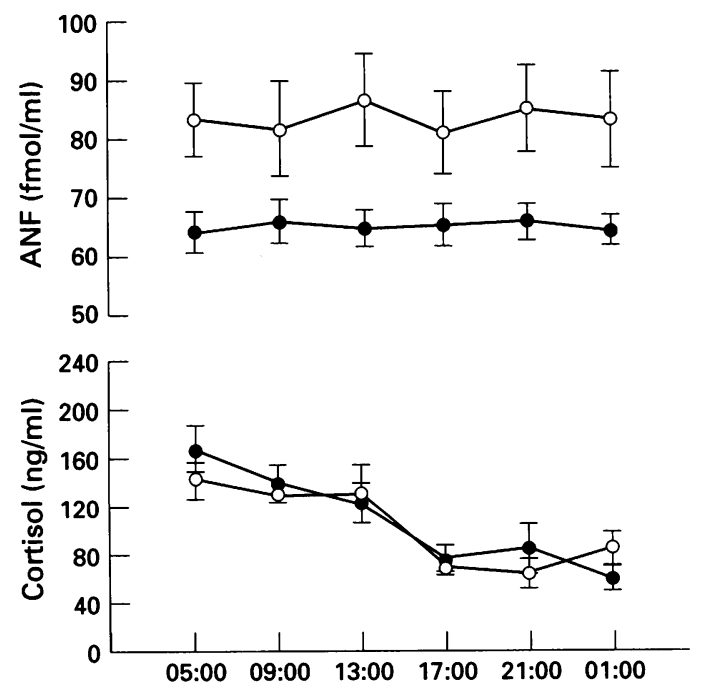

Circadian patterns of plasma atrial natriuretic factor (ANF) and cortisol during VVI pacing (empty circles) and DDD pacing (filled circles) in group B. Values are means with SEM from nine patients. 
employed. There was no significant difference between VVI and DDD pacing as regards the level of plasma cortisol.

\section{Discussion}

The principal focus of this study was the assessment of the levels of ANF over a 24 hour period during VVI and DDD pacing, to determine whether this peptide follows a circadian variation in paced patients. Previous studies have examined the same possibility in normal individuals without, however, establishing definitely whether ANF concentrations show the circadian distribution found in other hormones. ${ }^{3-6}$ A second aim of the study was the investigation of the difference in ANF concentrations under VVI and DDD pacing, not merely over a short time interval as in earlier studies, but over a complete 24 hour period for both pacing modes.

Our findings showed that there is no circadian pattern to the variations in ANF concentrations, either in patients who are paced for a short period (group A) or in those who are paced for a longer time (group B). The successive measurements of ANF in our study, under stable conditions, confirmed this, since none of the six measurements obtained in each pacing mode differed significantly from the others. In contrast, cortisol showed the same circadian pattern that it is known to occur in normal individuals. This difference supports the view that ANF has a pulsational pattern of secretion throughout the 24 hour period, without following any clear circadian variation.

The second observation of this study was that ANF concentrations were higher during VVI pacing than during DDD pacing, as confirmed by multiple measurements made over a 24 hour period. It is interesting that, in the patients who were paced for only a short period before the start of the measurements, this difference reached statistical significance only at $17.00,21.00$, and 01.00 , while in those who had been paced for two months the difference was significant for the whole 24 hour period. This can be attributed to the fact that a longer period of pacing, together with the haemodynamic changes caused by VVI pacing, may lead to the development of adaptive mechanisms in which ANF plays a part. In combination with the known role of the peptide in water and electrolyte homeostasis, this emphasises the "physiological" character of atrioventricular pacing, as well as proving that dual chamber pacing contributes to an improvement not only in cardiac haemodynamics but also in the neuroendocrine system.

1 Vardas $P$, Travill $C$, Williams $T$, Ingram $A$, Ligthman $S$ Sutton $R$. Effect of dual chamber pacing on raised plasma atrial natriuretic peptide concentrations in complete atrioventricular block. $B M \mathcal{F}$ 1988;296:94.

2 Stangl K, Weil J, Seitz K, Laule M, Gerzer R. Influence of atrioventricular synchrony on the plasma levels of atria natriuretic peptide (ANP) in patients with total atrioventricular block. Pace 1988;11:1176-81.

3 Haak T, Jungmann E, Schoffling K. 24-Hour variation in atrial natriuretic peptide. Lancet 1990;335:167-8.

4 Richards A, Tonolo G, Fraser R, Morton J, Leckie B, Ball S, et al. Diurnal change in plasma atrial natriuretic peptide et al. Diurnal change in plasma atrial nat.

5 Leppaluotto J, Ruskoaho H. Atrial natriuretic peptide, renin activity, aldosterone, urine volume and electrolytes renin activity, aldosterone, urine volume and electrolytes during a 24-h sleep-wak

6 Colantonio D, Pasqualetti P, Casale R, Natali G. Is atrial natriuretic peptide important in the circadian rhythm of natriuretic peptide important in the circadian rhyth
arterial blood pressure? $A m \mathcal{F}$ Cardiol 1988;63:1166. 